Article

\title{
Oral Clinical Manifestations of Neurofibromatosis Type 1 in Children and Adolescents
}

\author{
Rossella Santoro ${ }^{1,+}$, Claudia Santoro ${ }^{2,3,+}$, Francesca Loffredo ${ }^{1}$, Antonio Romano ${ }^{1}$, \\ Silverio Perrotta ${ }^{2} \mathbb{D}$, Rosario Serpico ${ }^{1}$, Dorina Lauritano ${ }^{4} \mathbb{( 1 )}$ and Alberta Lucchese ${ }^{1, *}$ \\ 1 Multidisciplinary Department of Medical-Surgical and Dental Specialties, \\ University of Campania “Luigi Vanvitelli”, Via Luigi de Crecchio 6, 80138 Naples, Italy; \\ rossella.santoro@unicampania.it (R.S.); francesca.loffredo1@studenti.unicampania.it (F.L.); \\ antonio.romano@unicampania.it (A.R.); rosario.serpico@unicampania.it (R.S.) \\ 2 Referral Centre of Neurofibromatosis, Department of Woman and Child, Specialistic and General Surgery, \\ University of Campania “Luigi Vanvitelli”, Via Luigi de Crecchio 2, 80138 Naples, Italy; \\ claudia.santoro@unicampania.it (C.S.); silverio.perrotta@unicampania.it (S.P.) \\ 3 Clinic of Child and Adolescent Neuropsychiatry, Department of Mental Health, \\ Physical and Preventive Medicine, University of Campania “Luigi Vanvitelli”, Via Luigi de Crecchio 2, \\ 80138 Naples, Italy \\ 4 Department of Medicine and Surgery, Centre of Neuroscience of Milan, University of Milano-Bicocca, \\ 20126 Milan, Italy; dorina.lauritano@unimib.it \\ * Correspondence: alberta.lucchese@unicampania.it; Fax: +39-081-5665477 \\ + These authors contributed equally to this work.
}

Received: 26 May 2020; Accepted: 5 July 2020; Published: 8 July 2020

\begin{abstract}
Background: Neurofibromatosis 1 (NF1) is an autosomal dominant genetic disorder. The expression of NF1 is extremely variable considering the broad spectrum of mutations affecting the gene(s) responsible for the pathology. Aim: To investigate the prevalence of oral manifestations in a group of children affected by neurofibromatosis type 1. Design: 100 pediatric patients, with genetically confirmed NF1 were enrolled in this study and matched to a total of 100 healthy children. Clinical examination was used to investigate: dental caries, dental abnormalities, periodontal health, neurofibromas, malocclusions, and enamel defects. Results: Mann Whitney's test concerning prevalence of dental caries resulted in a no significant difference between the two groups $(p=0.90)$; a significant difference was highlighted as regards the other kinds of manifestations as well: enamel defects $(p=0.01)$, neurofibromas $(p=0.0043)$ and poor oral hygiene $(p=0.0002)$ with a higher prevalence of these features in NF1 patients than healthy controls. Similar results come out, regarding dental abnormalities in which can observe a significant difference between shape anomalies $(p<0.001)$. Conclusion: According to data obtained from the present study, it can be stated that NF1-related oral manifestations can be detected during childhood and adolescence. In particular for neurofibromas, enamel defects, shape anomalies, and poor oral hygiene.
\end{abstract}

Keywords: neurofibromatosis type 1; children; oral; dental; DMFT

\section{Introduction}

Neurofibromatosis (NF) is a group of autosomal dominant genetic disorders [1], which can be divided into two subtypes, i.e., NF type 1 and NF type 2 (NF1 and NF2, respectively) [2]. Both can manifest as benign or malignant tumors of the central and peripheral nervous systems and multiple cutaneous lesions [1], although there are few features in common between the two subtypes because they are caused by mutations of different genes. Recent molecular genetics studies have discovered a marked delineation between the two variants of NF [3]. Diagnosis is made based on clinical 
features according to the criteria established by the National Institutes of Health (NIH) in 1987 [4]. NF1, which was previously known as Recklinghausen's disease [1], is the most common type, with an estimated incidence of 1 in 2500 to 3000 individuals independent of ethnicity, race and gender $[5,6]$. This disease is the consequence of a spectrum of mutations affecting the gene(s) located on chromosome 17q11.2 [3]; considering the broad spectrum of mutations, the expression of NF1 is extremely variable [6] and can show mild or several phenotypes. The classic manifestations of NF1 include café-au-lait macules (CALMs), axillary or inguinal freckling, iris Lisch nodules, neurofibroma and bone dysplasia. NF1 patients are prone to the development of brain tumours, especially optic pathway glioma (OPG) [7]. The other major features are neurological or cognitive impairment, scoliosis and abnormalities in the oral and maxillofacial region [8]. Oral manifestations are found in almost $72 \%$ of NF1 patients [9], and may include dental abnormalities, in particular impacted, displaced, supernumerary or missing teeth [8]; intrabony-neurofibromas; hypoplasia and deformity of the jaw; enlarged mandibular foramen; flat or missing gonial angle; coronoid notch deformity; pseudo elongation of the condylar processes; and soft tissues neurofibromas [10]. Gingival hyperplasia may also be associated with NF1 [11,12]. On the other hand, there is still some debate regarding whether caries represents a manifestation of this disease. This study was performed to evaluate the prevalence of oral manifestations and dental caries in children affected by NF1, as there is a paucity of data in the literature regarding oral findings in NF1 patients during childhood [10].

\section{Materials and Methods}

\subsection{Study Design and Patient Selection}

From September 2017 to December 2018 a case-control study was carried out at the University of Campania "Luigi Vanvitelli", Naples, Italy. The experimental group consisted of 100 patients ( 39 female and 61 males) with a genetically confirmed diagnosis of NF1 in care at the hospital, while the control group consisted of 100 healthy individuals ( 37 female and 63 male). The study was approved by the ethics committee of the University of Campania "Luigi Vanvitelli" (code 311/17). For both groups, informed consent was provided by their parents or guardians. Consecutive subjects in care at the hospital were selected before going through the inclusion/exclusion criteria. Patients in both groups had to comply with the inclusion criteria: to be between the ages of 2 and 18, not to be smokers, to have at least 10 teeth. Patients with decompensated systemic diseases like cardiac, inflammatory, hepatic, thyroid or renal alterations were excluded. Individuals with DM had to have HbA1c levels at $\leq 8 \%$ and take oral anti-diabetic therapy. The control group were healthy volunteers individuals, without diabetes or glucose intolerance, without decompensated systemic diseases like cardiac, inflammatory, hepatic, thyroid or renal alterations, matched by age and sex with the study group.

\subsection{Intraoral Examination}

The same procedure was applied for intraoral examination by the same two clinicians (RS \& FL); they visited all the subjects to standardize the data, screening the parameters as reported in Table 1 . The conditions reported in Table 1 were investigated. Table 1 shows and itemized the adopted protocol of the intraoral examination. Comparisons between the two groups were performed using statistical methods. 
Table 1. Intraoral examination chart of standardized protocol.

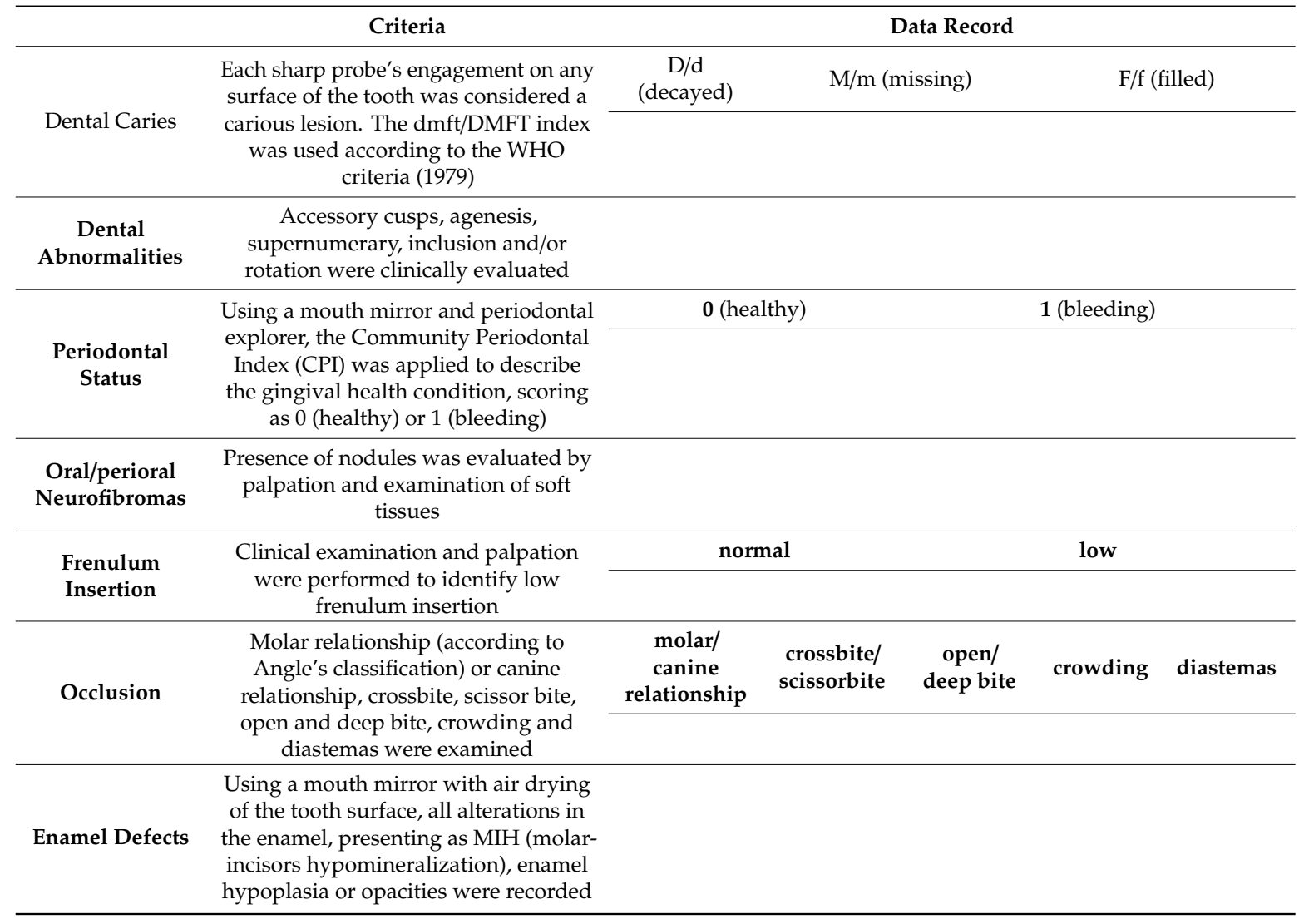

\subsection{Statistical Analysis}

Statistical analysis was performed using R software. Box plot show us the distribution of two samples (NF1 and control group). It is possible to see how DMFT of control group tend towards lower values than DMFT of NF1 patients. Outliers give us information about homogeneity of sample. Indeed, the two samples, given the presence of the outliers, aren't homogeneous. For this reason, Mann Whitney test was used to compare the average of the DMFT between the two groups. CHI-SQUARETEST was applied between the two groups for the other features.

\section{Results}

A total of 200 subjects were examined, consisting of 100 (39 females and 61 males, mean age of $9.18 \pm 4.3$ years) with NF1 and 100 (37 females and 63 males, mean age $9.12 \pm 4.2$ years) healthy subjects. Correlation between systemic manifestation of two groups are analyzed in Table 2. Comparisons between the oral conditions investigated in the two groups are reported in Table 3. In all analyses, $p<0.05$ was taken to indicate statistical significance (Table 3).

Table 2. Systemic manifestations detected in NF I and control groups.

\begin{tabular}{|c|c|c|c|c|c|c|c|c|c|}
\hline & CALMs & Astigmatism & Freckling & $\begin{array}{c}\text { Mental } \\
\text { Delay }\end{array}$ & $\begin{array}{c}\text { Lisch } \\
\text { Nodule }\end{array}$ & Neurofibroma & $\begin{array}{l}\text { Optical } \\
\text { Glioma }\end{array}$ & Myopia & Scoliosis \\
\hline $\begin{array}{c}\text { NF I } \\
\text { group }\end{array}$ & $75 \%$ & $5 \%$ & $14 \%$ & $4 \%$ & $12 \%$ & $15 \%$ & $5 \%$ & $7 \%$ & $13 \%$ \\
\hline $\begin{array}{l}\text { Control } \\
\text { group }\end{array}$ & - & - & - & - & - & - & - & - & - \\
\hline
\end{tabular}


Table 3. Statistical comparisons between the oral conditions investigated in the two groups analyzed.

\begin{tabular}{cccc}
\hline & NF1 Patients & Control Group & $p$ Value \\
\hline DMFT/dmft & 3.0 & 2.2 & 0.090 \\
\hline Enamel defects & $36 \%$ & $15 \%$ & 0.010 \\
\hline Malocclusion & $52 \%$ & $46 \%$ & 0.240 \\
\hline Frenulum with low insertion & $22 \%$ & $17 \%$ & \\
\hline Neurofibroma & $4 \%$ & 0 & 0.0043 \\
\hline Gingiva inflammation (CPI) & $17 \%$ & $15 \%$ & 0.0423 \\
\hline Poor Oral Hygiene & $41 \%$ & $16 \%$ & 0.0002 \\
\hline
\end{tabular}

Table 3 shows the number and the percentages of subjects with a particular manifestation versus control group: there were no significant differences between the two groups in $\mathrm{dmft} / \mathrm{DMFT}$ $(p=0.057)$, malocclusion $(p=0.2)$, frenulum insertion $(p=0.2)$ or gingiva inflammation CPI $(p=0.4)$. The Community Periodontal Index (CPI) was used to describe the gingival health condition, scored as 0 (healthy) or 1 (bleeding). Bleeding was present in 17 NF1 children and 15 children of the control group, showing a no statistically significant difference in the prevalence of gingiva inflammation.

Low frenulum insertion was present in 22 patients with NF1 and 17 subjects of the control group. Frenulum insertion was classified as low following the definition of Miller: "if the frenulum was unusually broad, and there was no apparent attached gingiva in the midline and the interdental papilla could be moved by stretching the frenulum" [13]. There was no statistically significant difference in the prevalence of frenulum insertion between the two groups: indeed, low frenulum insertion appears to be a frequent feature in all children, with no particular association with NF1.

There was, instead a significant difference about enamel defects $(p=0.0001)$, neurofibromas $(p=0.0043)$ and oral hygiene $(p=0.0002)$ with a higher prevalence of these features in NF1 patients than healthy controls.

Similar results come out, regarding dental abnormalities (Table 4) in which can be observe a significant difference between shape anomalies $(p<0.001)$ meanwhile for tooth rotation $(p=0.2)$, inclusion $(p=0.5)$ and agenesis $(p=0.5)$ doesn't observe a significant difference between the two groups.

Table 4. Statistical comparisons between single dental abnormalities.

\begin{tabular}{cccc}
\hline & NF1 Patients & Control Group & $p$ Value \\
\hline Shape Anomalies & $18 \%$ & $0 \%$ & $<0.001$ \\
\hline $\begin{array}{c}\text { Tooth } \\
\text { Inclusion }\end{array}$ & $2 \%$ & $1 \%$ & 0.5 \\
\hline $\begin{array}{c}\text { Tooth } \\
\text { Agenesis }\end{array}$ & $1 \%$ & $2 \%$ & 0.5 \\
\hline Tooth rotation & $1 \%$ & $4 \%$ & 0.2 \\
\hline
\end{tabular}

\section{Discussion}

There is some controversy in the literature regarding the oral manifestations of NF1. Neville et al. reported that oral manifestations are present in $4-7 \%$ of all cases [10], while other authors have reported much higher rates of oral findings [14,15]. D'Ambrosio concluded that $66 \%$ of NF1 patients showed at least one oral manifestation related to the disease [14]. Moreover, a recent study indicated that oral manifestations are present in 3.4-92\% of adult patients with NF1 [8]. Although there have been a number of reports in the literature regarding adult NF1 patients, few studies have evaluated the presence of oral manifestations in cases of paediatric NF1 [10]. 


\section{Dental caries}

The incidence of dental caries in NF1 patients $(\mathrm{dmft}=3.0)$ was higher than that in healthy controls $(\mathrm{dmft}=2.2)$ but without a statistically significant difference between two groups, in according with previous study [16]. Bardellini et al. reported similar results in a study comparing 50 NF1 patients to 50 patients without NF1 [10].

Other studies showed a significant difference between children with NF1 and healthy controls, in fact, a previous report by Tucker et al. and indicating an increased rate of dental caries in Canadian NF1 patients. However, this previous study was based on a questionnaire sent to families of children with NF1 and indicated an increased prevalence of caries in NF1 patients compared to unaffected siblings [17]. Tucker et al. explained the increase by referring to Lammert's study indicating that individuals with NF1 have a lower mean serum 25-hydroxyvitamin D3 (vitamin D) concentration than those without NF1 [18]. Data obtained by clinical examination in the present study build on the results of Tucker et al. as dental caries was detected not only by a survey but through an objective intraoral exam. However, recent studies yielded contradictory results regarding caries in NF1. For example, Visnapuu et al. reported that increased caries is not a typical feature of NF1 [19]. Indeed, their study showed a cumulative prevalence rate $(\mathrm{DMFT}>0)$ lower than that in the control group, probably due to increased efforts by parents to assist children with oral hygiene.

\section{Enamel defects}

Enamel defects represent an interesting topic for future studies. In fact, to our knowledge, only one previous study included enamel defects among dental abnormalities in NF1 patients [10].

In the present study, enamel defects appeared to be common in children with NF1 (36\%). It is possible that the manifestations of enamel defects were more visible in these children due to hyposalivation. However, as there is only one previous report in the literature regarding the association of this condition with NF [20], further studies are required to confirm the correlation.

Dental abnormalities

The most prevalent dental abnormalities in the present study were structural, particularly accessory cusps (18\%) (Figure 1).

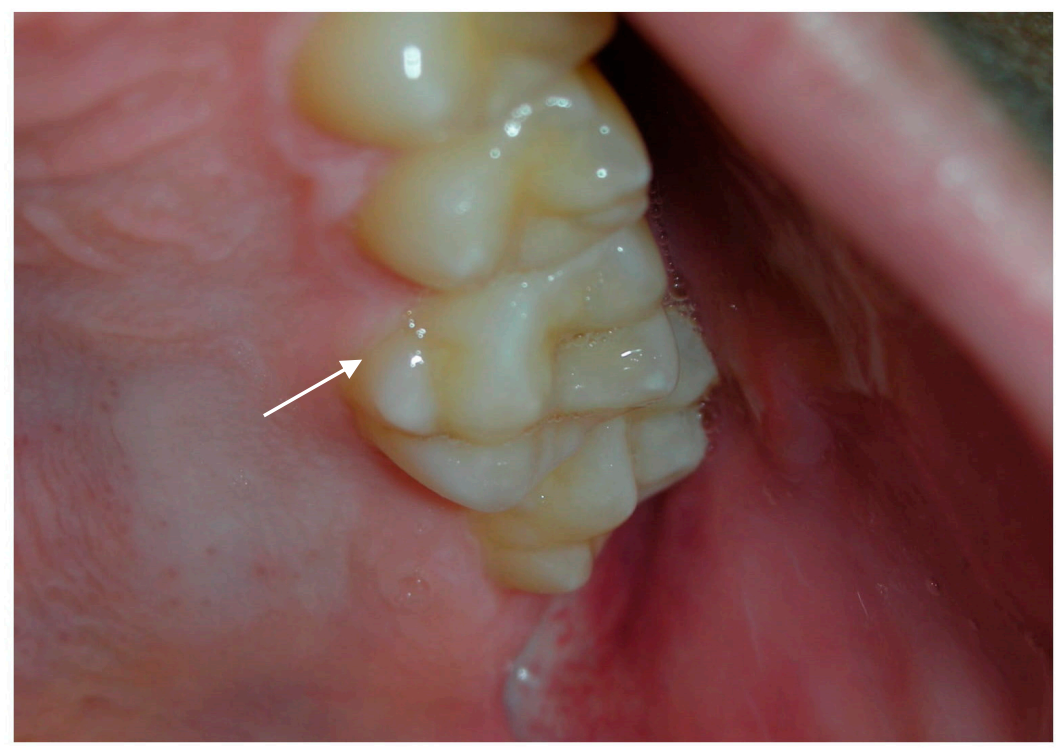

Figure 1. Accessory cusp, depicted by white arrow, found in a child affected by NF1.

Less common dental abnormalities included agenesis (1), inclusion (2), and/or rotation of the teeth (1). In a few patients, dental artefacts were found; in two cases these were associated with 
gingival hyperplasia due to the presence of neurofibroma or plexiform neurofibromas, as reported by Friederich et al. In two other studies, dental artefacts were accompanied by eruption difficulties [8]. For example, a dental artefact was found in association with a supernumerary tooth (8). In cases of dental abnormalities not associated with neurofibromas, a plausible explanation of these abnormalities could be consider as primitives, according to Bardellini et al.; however, unlike the present study, they found no significant difference between children affected by NF1 and healthy controls [10].

\section{Periodontal status}

In this study, both gingival health and oral hygiene were evaluated. Comparative analysis indicated a statistically significant difference between the two groups with regard to maintenance of adequate oral hygiene (NF1 $=41 \%$; Control Group $=16 \%$ ). There was no such difference in gingival health, indicating no relation of this parameter to the pathology of NF1 (NF1 $=17 \%$; Control Group $=15 \%$ ). These observations were in agreement with the report of Bardellini et al. [10]. Therefore, patients with NF1 appear to have poor oral hygiene, which may favour the progression of periodontal pathologies in adults affected by this disease. Two cases of gingival hyperplasia with a fibrous appearance were also recorded. Doufexis et al. reported that dental artefacts were associated with gingival hyperplasia [21].

\section{Neurofibromas}

Neurofibromas are benign complex tumours composed of all cell types that are found in the peripheral nerves. Development of neurofibromas on or around peripheral nerves is a distinctive symptom of NF1 [1]. The tongue is the most commonly involved site, and Shapiro et al. highlighted the presence of an enlarged tongue and fungiform papillae in NF1 [15]. This symptom occurs in $50 \%$ of cases, while gum neurofibromas occur in only $5 \%$ of cases [17]. There have been few previous reports concerning the presence of neurofibromas in paediatric patients, but the results of the present study indicated a significantly higher incidence of perioral neurofibromas in paediatric NF1 patients $(4 \%)$ compared to the control group (0), in contrast to Bardellini et al. [10]. Only two of the children included in the present study showed neurofibromas located on the lips (Figure 2).

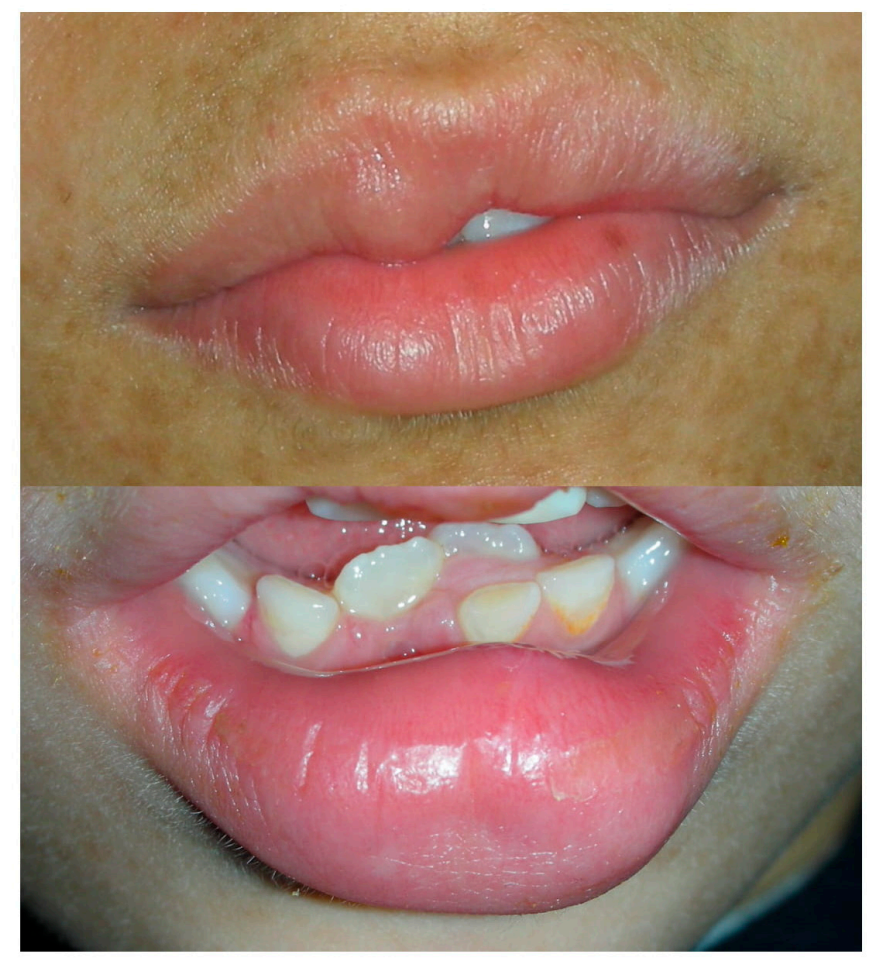

Figure 2. Multiple lips neurofibroma in NF I patient are showed. 
Another case showed multiple neurofibromas in the head and neck region, impacting the oral cavity (Figure 3).

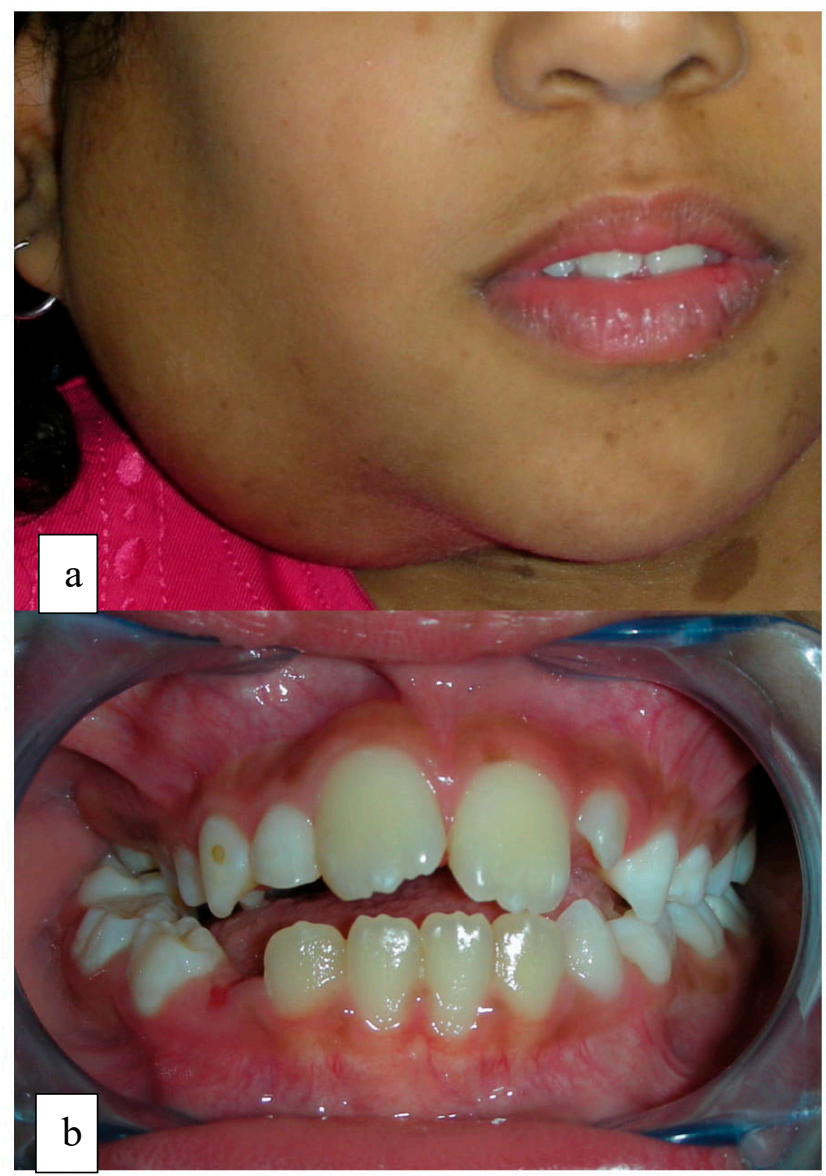

Figure 3. (a) Neurofibroma in the head and neck region; (b) class III malocclusion and posterior cross-bite in NF I patient.

In fact, one child showed gingival hyperplasia and relative malocclusion on the same side in relation to these neurofibromas.

Malocclusion

We found no significant difference in the occurrence of malocclusion between the study group (52\%) and controls (46\%). In clinical examinations, occlusion showed a relationship with the molars, canines, posterior cross bite, scissor bite, crowding and diastemas. These variables were considered as a single entity in the analyses in this study, although class III malocclusion, posterior cross bite and dental crowding tended to be more common in the study group compared to controls. Majorana A et al. carefully examined these orthodontic features and reported that class III malocclusion and posterior cross bite tended to be the most frequent conditions in NF1 patients [22]. Therefore, orthodontic screening should be performed in children with NF1 to allow early diagnosis of class III malocclusion.

\section{Frenulum insertion}

Given the frequency with which we observed frenulum insertion, we decided to report these findings. However, there was no statistically significant difference in the prevalence of frenulum insertion between the two groups. Low frenulum insertion appears to be a frequent feature in all children, with no particular association with NF1. 
In conclusion, according to data obtained from the present study, it can be stated that NF1-related oral manifestations can be detected during childhood and adolescence. In particular for neurofibromas, enamel defects, shape anomalies, and poor oral hygiene.

Dentists should be aware of the features that may be associated with this disease, particularly the high prevalence rates of caries and poor oral hygiene, dental abnormalities, enamel defects and a tendency towards class III malocclusion. Children with NF1 require special attention and dental care, in addition to specific treatment. Oral hygiene and oral health can be maintained by close collaboration between paediatricians, dentists and dental hygienists. It is important to increase the quality of life of children with NF1 to prevent the negative impact of the disease on oral health. Regular inspections of the oral condition in children with NF1 are necessary to prevent complications and manage therapy. Moreover, long-term follow-up of paediatric NF1 patients by dentists is required to prevent complications in adult life.

\section{Bullet Points}

1. Children with NF1 have oral manifestations such us dental abnormalities, enamel defects and a tendency towards class III

2. Oral Neurofibromas found in this study don't appear as frequent as could be expected.

3. Paediatric dentist should be aware to high prevalence of caries and poor oral hygiene in NF1 children in order to prevent further complications.

Author Contributions: A.L., R.S. (Rossella Santoro) and D.L. conceived the ideas; F.L., S.P. and A.R. collected the data; A.L., C.S. and R.S. (Rosario Serpico) analysed the data; F.L. and R.S. (Rossella Santoro) writing. All authors have read and agreed to the published version of the manuscript.

Funding: This research received no external funding.

Acknowledgments: The English in this document has been checked by at least two professional editors, both native speakers of English.

Conflicts of Interest: Authors declared no conflict of interest.

\section{References}

1. Gerber, P.A.; Antal, A.S.; Neumann, N.J.; Homey, B.; Matuschek, C.; Peiper, M.; Budach, W.; Bölke, E. Neurofibromatosis. Eur. J. Med. Res. 2009, 14, 102-105. [CrossRef] [PubMed]

2. Gutmann, D.H.; Aylsworth, A.; Carey, J.C.; Korf, B.; Marks, J.; Pyeritz, R.E.; Rubenstein, A.; Viskochil, D. The diagnostic evaluation and multidisciplinary management of neurofibromatosis 1 and neurofibromatosis 2. JAMA 1997, 278, 51-57. [CrossRef] [PubMed]

3. Cunha, K.S.; Barboza, E.P.; Dias, E.P.; Oliveira, F.M. Neurofibromatosis type I with periodontal manifestation. A case report and literature review. Br. Dent. J. 2004, 196, 457-460. [CrossRef] [PubMed]

4. Mulvihill, J.J.; Parry, D.M.; Sherman, J.L.; Pikus, A.; Kaiser-Kupfer, M.I.; Eldridge, R. NIH conference. Neurofibromatosis 1 (Recklinghausen disease) and neurofibromatosis 2 bilateral acoustic neurofibromatosis). An update. Ann. Intern. Med. 1990, 113, 39-52. [CrossRef] [PubMed]

5. Shen, M.H.; Harper, P.S.; Upadhyaya, M. Molecular genetics of neurofibromatosis type1 (NF1). J. Med. Genet. 1996, 33, 2-17. [CrossRef] [PubMed]

6. Williams, V.C.; Lucas, J.; Babcock, M.A.; Gutmann, D.H.; Korf, B.; Maria, B.L. Neurofibromatosis type 1 revisited. Pediatrics 2009, 123, 124-133. [CrossRef]

7. Santoro, C.; Bernardo, P.; Coppola, A.; Pugliese, U.; Cirillo, M.; Giugliano, T.; Piluso, G.; Cinalli, G.; Striano, S.; Bravaccio, C.; et al. Seizures in children with neurofibromatosis type 1: Is neurofibromatosis type 1 enough? Ital. J. Pediatr. 2018, 44, 41. [CrossRef]

8. Friedrich, R.E.; Giese, M.; Schmelzle, R.; Mautner, V.F.; Scheuer, H.A. Jaw malformations plus displacement and numerical aberrations of teeth in neurofibromatosis type 1: A descriptive analysis of 48 patients based on panoramic radiographs and oral findings. J. Craniomaxillofac. Surg. 2003, 31, 1-9. [CrossRef]

9. Geist, J.R.; Gander, D.L.; Stefanac, S.J. Oral manifestations of neurofibromatosis types I and II. Oral Surg. Oral Med. Oral Pathol. 1992, 73, 376-382. [CrossRef] 
10. Bardellini, E.; Amadori, F.; Flocchini, P.; Conti, G.; Piana, G.; Majorana, A. Oral findings in 50 children with neurofibromatosis type 1. A case control study. Eur. J. Paediatr. Dent. 2011, 12, 256-260.

11. Mahajan, A.; Dixit, J.; Bhardwaj, A. Gingival enlargement in neurofibromatosis type1: A case report and literature review. J. Contemp. Dent. Pract. 2010, 11, 057-063. [PubMed]

12. Bekisz, O.; Darimont, F.; Rompen, E.H. Diffuse but unilateral gingival enlargement associated with von Recklinghausen neurofibromatosis: A case report. J. Clin. Periodontol. 2000, 27, 361-365. [CrossRef] [PubMed]

13. Miller, P.D., Jr. The frenectomy combined with a laterally positioned pedicle graft. Functional and esthetic considerations. J. Periodontol. 1985, 56, 102-106. [CrossRef] [PubMed]

14. D'Ambrosio, J.A.; Langlais, R.P.; Young, R.S. Jaw and skull changes in neurofibromatosis. Oral Surg. Oral Med. Oral Pathol. 1988, 66, 391-396. [CrossRef]

15. Shapiro, S.D.; Abramovitch, K.; Van Dis, M.L.; Skoczylas, L.J.; Langlais, R.P.; Jorgenson, R.J.; Young, R.S.; Riccardi, V.M. Neurofibromatosis: Oral and radiographic manifestations. Oral Surg. Oral Med. Oral Pathol. 1984, 58, 493-498. [CrossRef]

16. Tsang, E.S.; Birch, P.; Friedman, J.M.; Johnston, D.; Tucker, T.; Armstrong, L. Prevalence of dental caries in children with neurofibromatosis 1. Clin. Oral Investig. 2010, 14, 479-480. [CrossRef]

17. Tucker, T.; Birch, P.; Savoy, D.M.; Friedman, J.M. Increased dental caries in people with neurofibromatosis 1. Clin Genet. 2007, 72, 524-527. [CrossRef]

18. Lammert, M.; Friedman, J.M.; Roth, H.J.; Friedrich, R.E.; Kluwe, L.; Atkins, D.; Schooler, T.; Mautner, V.F. Vitamin D deficiency associated with number of neurofibromas in neurofibromatosis 1. J. Med. Genet. 2006, 43, 810-813. [CrossRef]

19. Visnapuu, V.; Peltonen, S.; Tammisalo, T.; Peltonen, J.; Happonen, R.P. Radiographic findings in the jaws of patients with neurofibromatosis 1. J. Oral Maxillofac. Surg. 2012, 70, 1351-1357. [CrossRef]

20. Cunha, K.S.; Rozza-de-Menezes, R.E.; Luna, E.B.; Almeida, L.M.; Pontes, R.R.; Almeida, P.N.; de Aguiar, L.V.; Dias, E.P. High prevalence of hyposalivation in individuals with neurofibromatosis 1: A case-control study. Orphanet J. Rare Dis. 2015, 10, 24. [CrossRef]

21. Doufexi, A.; Mina, M.; Ioannidou, E. Gingival overgrowth in children: Epidemiology, pathogenesis, and complications. A literature review. J. Periodontol. 2005, 76, 3-10. [CrossRef] [PubMed]

22. Majorana, A.; Bardellini, E.; Amadori, F.; Conti, G.; Polimeni, A. Timetable for oralprevention in childhood-developing dentition and oral habits: A current opinion. Prog. Orthod. 2015, 16, 39. [CrossRef] [PubMed]

(C) 2020 by the authors. Licensee MDPI, Basel, Switzerland. This article is an open access article distributed under the terms and conditions of the Creative Commons Attribution (CC BY) license (http://creativecommons.org/licenses/by/4.0/). 